\title{
AIDS-associated plasmablastic lymphoma presenting as a poorly differentiated esophageal tumor: a diagnostic dilemma D Mani ${ }^{1,2}$, D Guinee $\mathrm{Jr}^{3}$ and D Aboulafia*4
}

\author{
Address: ${ }^{1}$ Division of Hematology and Oncology, Virginia Mason Medical Center, Seattle, Washington, USA, ${ }^{2}$ Internal Medicine Residency, \\ Spokane Medical Centers, Spokane, Washington, USA, ${ }^{3}$ Division of Pathology, Virginia Mason Medical Center, Seattle, Washington, USA and \\ ${ }^{4}$ Division of Hematology, University Of Washington, Seattle, Washington, USA \\ * Corresponding author
}

\author{
from I I th International Conference on Malignancies in AIDS and Other Acquired Immunodeficiencies (ICMAOI): Basic, Epidemiologic, and Clinical \\ Research \\ Bethesda, MD, USA. 6-7 October 2008 \\ Published: 17 June 2009 \\ Infectious Agents and Cancer 2009, 4(Suppl 2):P2 doi:I0.II 86/I750-9378-4-S2-P2
}

This abstract is available from: http://www.infectagentscancer.com/content/4/S2/P2

(c) 2009 Mani et al; licensee BioMed Central Ltd.

Plasmablastic lymphoma (PBL) is a rare form of diffuse large B-cell lymphoma characterized by weak or absent expression of conventional B-cell markers and strong expression of plasma cell markers. It is strongly associated with HIV and Epstein Barr Virus (EBV) infection, and shows an unusual tropism to the oral cavity. Herein we describe a patient with AIDS who presented with weight loss and dysphagia owing to a large ulcerative gastroesophageal mass. His radiographic and endoscopic findings and long history of cigarette consumption suggested esophageal carcinoma. The patient had a history of multidrug resistant HIV and had been placed on salvage HAART (tenofovir, emtricitabine, ritonavir and atazanavir). Despite recent weight loss of 15 pounds, his CD4+ count had improved from 50 to 180 cells/iL and his HIV viral load, previously $>100,000$ copies $/ \mathrm{ml}$ was now undetectable. Biopsy demonstrated a poorly differentiated tumor staining negatively to routine lymphoid markers including CD20. However, gene rearrangement studies confirmed a B-cell process and a more detailed immunohistochemistry analysis revealed the cells expressing CD138 (plasma cell antigen). In situ hybridization studies were positive for EBV but negative for Human Herpes Virus type-8. These findings were diagnostic of PBL and the patient was treated with combination chemotherapy consisting of liposomal doxorubicin, cyclophosphamide and etoposide. After six cycles of chemotherapy, his dysphagia and weight loss resolved and a positron emission tomogram showed complete res- olution of the esophagogastric mass. The patient remains in complete remission 12 months later.

Our report underscores the importance of a broad array of viral and molecular studies needed to establish the diagnosis of PBL, which may mimic a variety of entities including carcinoma, melanoma, Kaposi's sarcoma, plasmacytoma and primary effusion lymphoma. Furthermore, our patient's diagnosis of lymphoma within 1 year of HAART initiation, and in the context of a rapidly improving CD4+ cell count and non-detectable HIV viral load may represent a case of immune reconstitution syndrome associated Non-Hodgkin's lymphoma. 